\title{
Fuzzy binary patterns for uncertainty-aware texture representation
}

\author{
E.G. Keramidas ${ }^{+}$, D.K. Iakovidis ${ }^{*}$ and D. Maroulis ${ }^{+}$ \\ + Dept. of Informatics and Telecommunications, University of Athens, Panepistimiopolis, GR-15784, Athens, Greece \\ * Technological Educational Institute of Lamia, Dept. of Informatics and Computer Technology, GR 35100, Greece
}

Received 8th Aug 2010; accepted 7th Oct 2011

\begin{abstract}
A wide range of pattern recognition applications have been based on the Local Binary Pattern (LBP) representation of textures, including texture segmentation, face detection, and biomedical image analysis. The interest of the research community in the LBP texture representation gave rise to plenty of LBP and other Binary Pattern (BP)-based variations. However, noise sensitivity is still a major concern to their applicability on the analysis of real world images. To cope with this problem we propose a generic, uncertainty-aware methodology for the derivation of Fuzzy BP (FBP) texture models. The proposed methodology assumes that a local neighbourhood can be partially characterized by more than one binary patterns due to noise-originated uncertainty in the pixel values. The texture discrimination capability of four representative FBP-based approaches has been evaluated on the basis of comprehensive classification experiments on reference datasets of natural textures under various types and levels of additive noise. The results reveal that the FBP-based approaches lead to consistent improvement in texture classification as compared with the original BP-based approaches for various degrees of uncertainty. The performance advantage of the FBP-based approaches has been also validated by unsupervised segmentation of natural scenes.
\end{abstract}

Key Words: Texture, Fuzzy Sets, Noise, Binary Patterns.

\section{Introduction}

Texture analysis concerns a considerable range of applications such as remote sensing, biomedical image processing, visual inspection, object discrimination, terrain delimitation and image classification [1-4]. Since the early seventies a variety of textural feature extraction approaches have been proposed $[5,6]$. The Local Binary Pattern (LBP) approach [7], which is based on the concept of binary patterns (BP) for the representation of texture, has been widely adopted because it is simple, yet effective in describing the local spatial structure of an image. The LBP approach has been extended and combined with other approaches resulting in a variety of texture representation schemes suitable for different image analysis tasks. Typical examples include LBP extensions featuring scale invariance [8], rotation invariance [9-10], combination of

Correspondence to: <e.keramidas@gmail.com>

Recommended for acceptance by $<$ Thomas Breuel $>$

ELCVIA ISSN: 1577-5097

Published by Computer Vision Center / Universitat Autonoma de Barcelona, Barcelona, Spain 
inter and intra spatial structure of the LBP patterns [11], and fusion of micro LBP and macro Gabor features [12].

The LBP texture representation has drawn a lot of interest from the research community, resulting in plenty of variations. Several approaches based on the BP model have been investigated. Such an approach has been proposed for the estimation of a local contrast measure [7]. The LBP/C approach, which is based on the joint distribution of the LBP codes and the local contrast measures, has shown resistance to variations of the illumination and has been used to enhance the discrimination ability of the original LBP approach [7].

Another variation of the LBP, the Local Edge Patterns (LEP) approach, has been proposed for image segmentation in [13]. It describes the spatial structure of the local texture according to the spatial arrangement of edge pixels. In the same spirit, Hafiane et al. [14] proposed the Median Binary Pattern (MBP) which is intensity-shift invariant. According to this approach, the texture primitives are determined by localized thresholding against the local median. In a subsequent study, a hashed version of the MBP to a binary chain or equivalence class has been evaluated, resulting in a resolution and rotation invariant MBP (MBP ROT) texture descriptor [15].

The applications of the BP-based approaches in pattern recognition are numerous (Table 1). One of the first studies on visual inspection [16] has shown that the LBP features can be successfully used for surface defect detection. Later, in [17], BP-based features have been used for wood quality discrimination, and more recently such features have also applied on automatic defect detection [18][19] and remote sensing [20]. A variety of studies have shown that the BP-based feature extraction approaches are suitable for content-based image retrieval [21-22], whereas recently the LBP approach has been adopted in a discriminative model for image ranking from text queries [23]. Moreover, in the area of face recognition the LBP is recognized as a highly efficient texture representation approach. For example, it has been used for invariant face recognition [24][25], face authentication [26], and the recognition of facial expressions [27]. Excellent results have also been obtained from its application on the biomedical domain, including video endoscopy [28], the classification of protein images [29], and the computer aided neuroblasoma prognosis system [30]. In the area of motion analysis the application of BP-based approaches has been investigated for underwater image matching [31], modelling and detection of moving objects [32], and object tracking [33].

\begin{tabular}{lc}
\hline Application & Reported in \\
\hline Visual Inspection & {$[17-19]$} \\
Remote Sensing & {$[20]$} \\
Image \& Video & {$[21-23]$} \\
Face Recognition & {$[24-27]$} \\
Biomedical Imaging & {$[28-30]$} \\
Motion Analysis & {$[31-33]$} \\
\hline
\end{tabular}

Table 1: Applications of BP-based approaches in pattern recognition.

A major drawback of the current BP-based approaches is that the binary patterns are extracted via pixelwise comparisons. This makes them sensitive to noise and to small variations in the pixel values, thus limiting its real world applicability. Real world images acquired with conventional techniques are subject to various sources of noise introducing uncertainty in their pixel values. Principal noise sources include sensor instability and transmission channel interferences [34].

Recent studies indicate that the application of the fuzzy sets theory on the extraction of texture spectrum features [35] and their efficient successors, the LBP features, could possibly enhance their robustness to noise [36-39]. However, these studies can only be considered as preliminary since they include only a limited experimental evaluation. In this paper we propose a generic, uncertainty-aware methodology for the derivation of Fuzzy BP (FBP) texture models. The proposed methodology aims to provide improved BP texture representations robust to the presence of noise. Its application is investigated through fuzzification of a variety of $\mathrm{BP}$ approaches, including the LBP, LBP/C, LEP and the MBP. The improved texture 
representations obtained are validated with a comprehensive and systematic experimental study on standard collections of textures and natural scenes.

The rest of this paper is organized in four sections. Section 2 presents the BP-based texture representation approach using a generic formulation based on crisp sets. Section 3 presents the proposed methodology for the derivation of the FBP texture model, and the results from its comprehensive experimental evaluation are presented in section 4 . The last section summarises the conclusions derived from this study.

\section{Crisp Binary Patterns}

The BP texture model is based on the crisp pairwise comparison of pixel grey-levels. Each pixel $p_{x}$ in a square neighborhood is characterized by a comparison of its grey-level $g_{x}$ with a reference grey-level $g_{\text {reference }}$ that is common for all pixels in that neighbourhood (Fig. 1). Thus, two crisp sets of pixels are defined. Let $B$ be the set of all pixels $p_{x}$ of the neighborhood with grey-level $g_{x}$ greater than or equal to $g_{\text {reference }}$, and $S$ be the set of all pixels $p_{x}$ with grey-level smaller than $g_{\text {reference }}$. Then the set $B$ can be expressed by the following equation:

$$
B \equiv\left\{p_{x} \mid L_{B}(x)\right\}
$$

where $L_{B}(x)$ is a predicate defined as $g_{x} \geq g_{\text {reference. }}$. Hence the set $S$ is the complement of $B\left(S \equiv B^{C}\right)$ relative to the universal set $H$ of all pixels of the current neighborhood.

A characteristic function $m_{B}(\mathrm{x})$ can be utilized to mathematically describe the crisp set $B$ as follows:

$$
m_{B}(x)= \begin{cases}1 & \text { if } p_{x} \in B \\ 0 & \text { if } p_{x} \notin B\end{cases}
$$

Based on these binary values, for each n-pixels neighborhood, a unique BP code can be computed:

$$
B P_{\text {CODE }}=\sum_{x=0}^{k-1} d_{x} \cdot w_{x}
$$

where $k \in(0, n]$ is the number of neighborhood pixels participating in the $B P_{\text {code }}$ computation, $d_{x}=m_{B}(x)$ and $w_{x}=2^{x}$. Thus, each local neighborhood is characterized by a single BP code, out of $2^{k}$ possible codes (Fig. 1).

For a given image region, a histogram counts the occurrences of the BP codes for all the local neighborhoods within the region. This histogram forms a feature vector, representing the texture of that region.

\begin{tabular}{|l|l|l|}
\hline$g_{0}$ & $g_{1}$ & $g_{2}$ \\
\hline$g_{7}$ & $g_{8}$ & $g_{3}$ \\
\hline$g_{6}$ & $g_{5}$ & $g_{4}$ \\
\hline
\end{tabular}

(a)

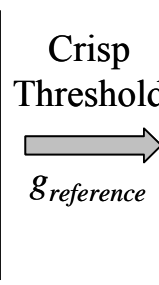

Figure 1: BP computation scheme for a $3 \times 3$-pixel neighbourhood. (a) Grey-levels of a $3 \times 3$ neighbourhood of pixels. Entrywise multiplication of (b) binary values matrix and (c) binomial weights matrix. (d) BP code. 


\section{$3 \quad$ Fuzzy Binary Patterns}

The crisp BP approach described in the previous section is based on a hard thresholding scheme defined by the predicate $L_{B}(x)$. This makes the BP texture representation scheme vulnerable to the grey-level uncertainty that is inherently present in digital images. By incorporating fuzzy logic to the computation of the binary patterns, an uncertainty-aware representation of local texture can be obtained, providing improved discrimination of textures in the presence of uncertainty.

Fuzzy logic was introduced in [40] as a multivalued logic, that allows intermediate values to be defined between conventional evaluations like true/false, and high/low. In the context of texture representation, the crisp sets $B$ and $S$ defined in the previous section, can be re-defined as two fuzzy sets $\widetilde{B}$ and $\widetilde{S}$, of pixels $p_{x}$ with grey-levels falling roughly in $\left[g_{\text {reference }}, g_{\max }\right]$ and $\left[0, g_{\text {reference }}\right)$, respectively. The value of $g_{\max }$ is the maximum grey-level that can be given to a pixel.

Formally, the fuzzy set $\widetilde{B}$ can be expressed as a set of ordered pairs

$$
\widetilde{B} \equiv\left\{\left\langle p_{x}, \mu_{\widetilde{B}}(x)\right\rangle \mid x \in H\right\}
$$

where $\mu_{\widetilde{B}}(x)$ is a membership function for the fuzzy set $\widetilde{B}$ and $H=\{0,1,2, \ldots, n-1\}$ for a $n$-pixel neighborhood. The membership function $\mu_{\widetilde{B}}(x)$ relates a real membership grade from the closed interval $[0,1]$, to each $p_{x}$, representing the degree to which $p_{x}$ belongs to fuzzy set $\widetilde{B}$. Accordingly, the fuzzy set $\widetilde{S}$ can be expressed as a set of ordered pairs

$$
\widetilde{S} \equiv\left\{\left\langle p_{x}, \mu_{\widetilde{S}}(x)\right\rangle \mid x \in H\right\}
$$

where $\mu_{\widetilde{S}}(\mathrm{x})$ is a membership function for fuzzy set $\widetilde{S}$.

The membership function $\mu_{\widetilde{B}}(x)$, for the fuzzy set $\widetilde{B}$, can be defined as the following increasing function:

$$
\mu_{\tilde{B}}(x)= \begin{cases}1 & \text { if } g_{x}-g_{\text {reference }} \geq T \\ \frac{T+g_{x}-g_{\text {reference }}}{2 \cdot T} & \text { if }\left|g_{x}-g_{\text {reference }}\right|<T, T \neq 0 \\ 0 & \text { if } g_{x}-g_{\text {reference }} \leq-T, T \neq 0 \\ 0 & \text { if } g_{x}-g_{\text {reference }}<T, \quad T=0\end{cases}
$$

Similarly, the membership function $\mu_{\widetilde{S}}(\mathrm{x})$ can be defined as:

$$
\mu_{\widetilde{S}}(\mathrm{x})=1-\mu_{\widetilde{B}}(\mathrm{x})
$$

For both $\mu_{\widetilde{B}}(x)$ and $\mu_{\widetilde{S}}(\mathrm{x}), T \in\left[0, g_{\max }\right]$ represents a parameter that controls the degree of fuzziness.

According to the proposed FBP approach, a neighbourhood of $\mathrm{n}$ pixels can be characterized by more than one ordered pairs of BP codes and CBP values. A CBP value expresses the contribution of a BP code to the BP histogram or in other words the degree to which a BP code characterizes a neighbourhood. It is estimated from the membership functions $\mu_{\widetilde{B}}(x)$ and $\mu_{\widetilde{S}}(\mathrm{x})$ as follows:

$$
C_{B P}=\prod_{\substack{X=0 \\ Z \in\{\tilde{B}, \widetilde{S}\}}}^{k-1} \mu_{Z}(x)
$$

where $k \in(0, n]$ is again the number of neighborhood pixels participating in the BPcode computation. 


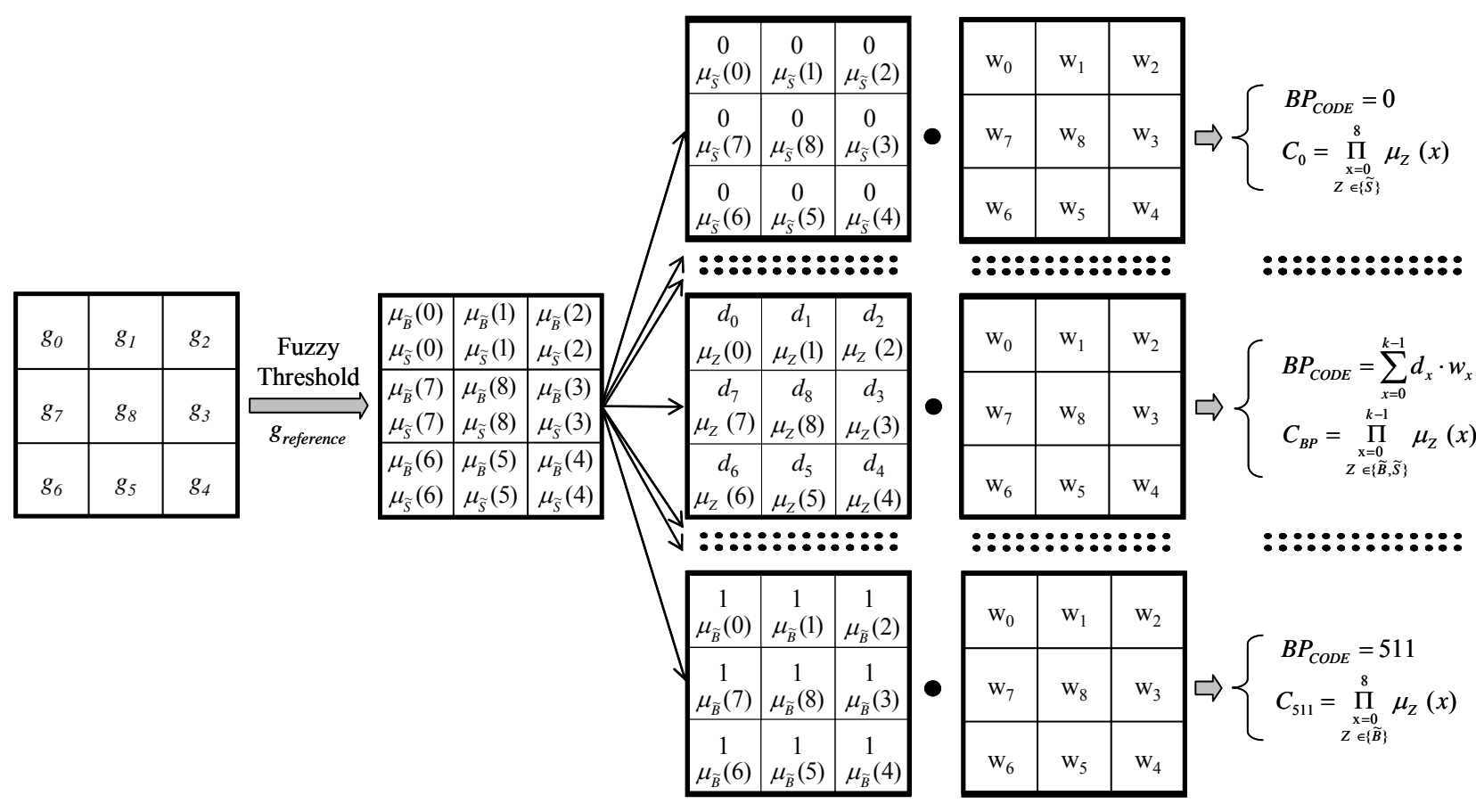

(a) (c) (d)

Figure 2: FBP computation scheme for a 3×3-pixel neighbourhood. (a) Grey-levels of the neighbourhood

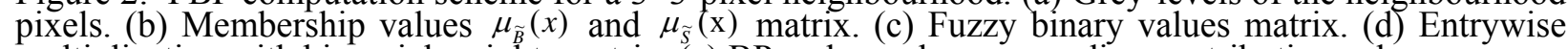
multiplication with binomial weights matrix. (e) BP codes and corresponding contribution values.

The FBP computation scheme for $n=9$ pixels neighbourhood and $k=9$ is illustrated in Fig. 2 . To compute a BP code through Eq. 3 the values of $d_{x}$ should be defined as follows:

$$
d_{x}=\left\{\begin{array}{l}
1 \quad \text { if } Z \equiv \widetilde{B} \\
0 \quad \text { if } Z \equiv \widetilde{S}
\end{array}\right.
$$

Thus each neighbourhood contributes to more than one bin in the FBP histogram. The total contribution $C_{\text {Total }}$ of an n-pixel neighbourhood to the histogram of the BP codes is always equal to one.

$$
C_{\text {Total }}=\sum_{B P=0}^{2^{k}-1} C_{B P}=1
$$

It can be noticed that the proposed FBP representation reduces to the crisp BP representation for membership values restricted to $\{0,1\}$. An example of the FBP computation scheme for a real $3 \times 3$-pixel neighborhood is shown in Fig.3. 


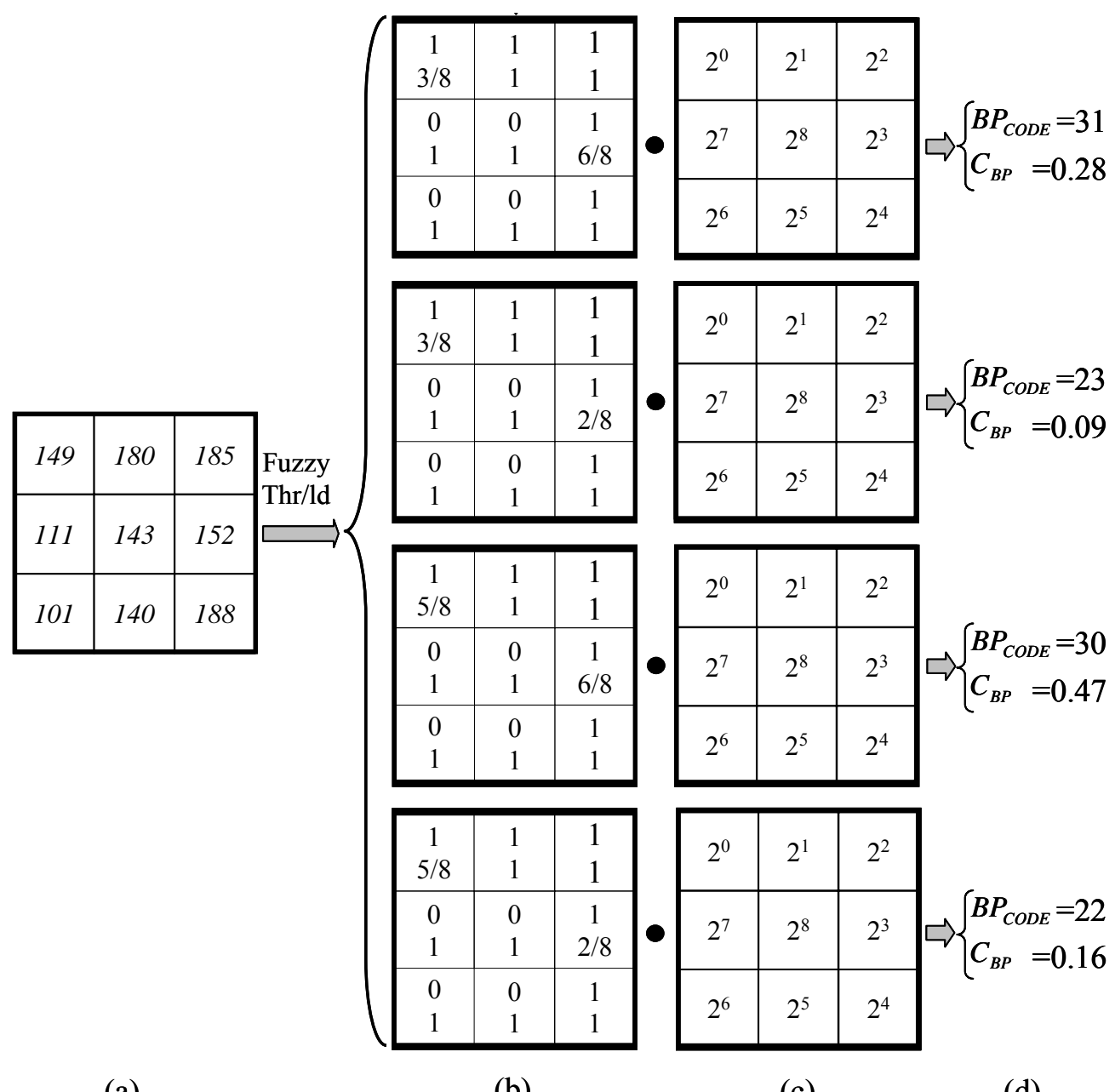

(a)

(b)

(c)

(d)

Figure 3: Example of FBP computation scheme for a 3×3-pixel neighbourhood with $g_{\text {reffernce }}=150$ and $T=4$. (a) Grey-levels of the neighbourhood pixels. (b) Fuzzy binary matrix with $d_{x}$ and $\mu_{z}$ (x) values. (c) Binomial weights matrix. (d) BP codes and corresponding contribution values.

In the following, we apply the proposed FBP model for the fuzzification of four representative BP-based methodologies.

\subsection{Fuzzy local binary patterns}

In the original crisp LBP texture model [7], $g_{\text {reference }}$ is the grey-level $g_{8}$ of the central pixel $p_{8}$ in the $3 \times 3$ local neighbourhood (Fig. 1). For each local neighbourhood an LBP code can be computed by Eq. (3) for $k=8$. Therefore, each $3 \times 3$-pixel neighborhood is represented by one out of $2^{8}=256$ possible LBP codes.

A fuzzy LBP (FLBP) representation can be obtained, for a reference value $g_{\text {reference }}$ equal to the grey-level of the central pixel $p_{8}$, from Eqs. (3) and (9). The corresponding contributions $C_{L B P}$ of the LBP codes in the fuzzy LBP histogram can defined through Eq. (8), for $k=8$. For a $3 \times 3$-pixel neighborhood the computational cost of the FLBP approach is, in the worst case, $2^{8}=256$ times the computational cost of the original crisp LBP. 


\subsection{Fuzzy local contrast for fuzzy local binary patterns}

A BP-based contrast measure has been proposed as a complementary feature, enhancing the LBP texture representation in [7]. For each LBP code a contrast measure can be computed as follows:

$$
C=\operatorname{avg}\left(g_{i}\right)-\operatorname{avg}\left(g_{j}\right)
$$

where $g_{i}$ is the grey-level of every pixel $p_{i}$ of the local neighbourhood that belongs to the crisp set $B$, and $g_{j}$ is the grey-level of every pixel $p_{j}$ of the neighbourhood that belongs to the crisp set $S$. The resulting LBP/C feature vector characterizing a neighbourhood of pixels in this case is a two dimensional histogram of the joint LBP-contrast occurrences.

The fuzzy analogous to the crisp LBP/C approach, FLBP/FC, can be obtained by computing the FLBP codes as described in subsection 3.1. The fuzzy contrast value for each FLBP code from Eq. (11), where $g_{i}$ is the grey-level of every pixel $p_{i}$ of the neighbourhood that belongs to fuzzy set $\widetilde{B}$, and $g_{j}$, is the grey-level of every pixel $p_{j}$ of the neighbourhood that belongs to fuzzy set $\widetilde{S}$. The feature vector characterizing a neighbourhood is now a two dimensional fuzzy histogram of the joint FLBP-fuzzy contrast occurrences. In the case of FLBP/FC approach for a 3×3-pixel neighbourhood the computational cost is, in the worst case scenario, $2^{8} \times G_{\text {contrast }}$ times the computational cost of the original crisp LBP, where $G_{\text {contrast }}$ is the number of bins of the contrast's histogram.

\subsection{Fuzzy median binary patterns}

The Median Binary Pattern (MBP) approach [14] is based on binary patterns computed by comparing the pixels of a neighborhood against their median value. The MBP is computed from Eq. (3), with a $g_{\text {reference }}$ that is equal to the median grey-level value of the local neighbourhood. All the 9 pixels of the $3 \times 3$-pixel neighbourhood are compared with the local median. Thus there are $2^{9}=512$ possible MBP codes to represent a 3×3-pixel neighborhood.

A Fuzzy Median Binary Pattern (FMBP) code is computed from Eqs. (3) and (9), and the corresponding contribution $C_{M B P}$ from Eq. (8), for $k=9$ and a $g_{\text {reference }}$ that is equal to the median grey-level value of the local neighborhood. For a $3 \times 3$-pixel neighborhood in the worst case scenario, the computational cost of the FMBP approach is, $2^{9}=512$ times the computational cost of the original crisp MBP, for a $3 \times 3$-pixel neighbourhood.

\subsection{Fuzzy local edge patterns}

The computation of Local Edge Patterns (LEP) presupposes the generation of an edge image [13]. The edge image is obtained by applying the Sobel operator [41] to a grey-level image. From the generated edge image the LEP operator is obtained by Eq. (3), for $k=9$ and $g_{\text {reference }}=150$ as suggested in [13]. Since the central pixel is included in this filtering process, there will be $2^{9}=512$ possible LEP codes to represent a $3 \times 3$-pixel neighborhood. The computational cost of the LEP approach is $\mathrm{O}(n)$, for an image with $n$ pixels.

The fuzzy analogous to the crisp LEP approach (FLEP), can be derived from an edge image, with the LEP codes computed from Eq. (3) and (9), and each corresponding contribution $C_{F L E P}$ from Eq. (8), for $k=9$ and $g_{\text {reference }}=150$. The FLEP approach, in the worst case scenario, has a computational cost of $2^{9}=512$ times the computational cost of the original crisp LEP, which results in a computational complexity of $\mathrm{O}(n)$.

\section{Experimental evaluation}

More than 2,800 experiments were conducted for the evaluation of the FBP-based approaches using three reference datasets of natural textures. These include textures from the Outex13 [42], the Brodatz [43], and the Vistex [44] image collections. For each dataset the original textures were divided into two sets by using a 
checkerboard pattern. Half of the resulted images constituted the training set, starting from the upper left, whereas the other half constituted the testing set. This resulted in two independent equicardinal, balanced sets, for the training and the testing classification phases.

The $k$-Nearest Neighbor ( $k$-NN) classifier [45] was used because it is a simple, non-parametric and proved competitive on a number of pattern recognition tasks [46]. Considering that the computed feature vectors are statistical distributions, the intersection of the distributions [47] was selected as an effective distance measure in the $k-\mathrm{NN}$ classifier.

The FBP-based approaches presented in paragraphs 3.1 to 3.4, were evaluated in comparison with the conventional BP-based approaches, on the original and on noise-degraded image datasets. In order to assess their performance in the presence of uncertainty, the additive noise model was considered. This type of noise is the most commonly occurring in real-world images which are subject to thermal noise introduced by photo-electronic sensors and transmission channels [48]. To this end three additive noise models, the uniform, the white Gaussian, and the exponential, were applied on the image dataset. The level of noise added was measured in terms of signal-to-noise ratio (SNR) ranging between 6 and $12 \mathrm{~dB}$ mean SNR (Fig. 4).
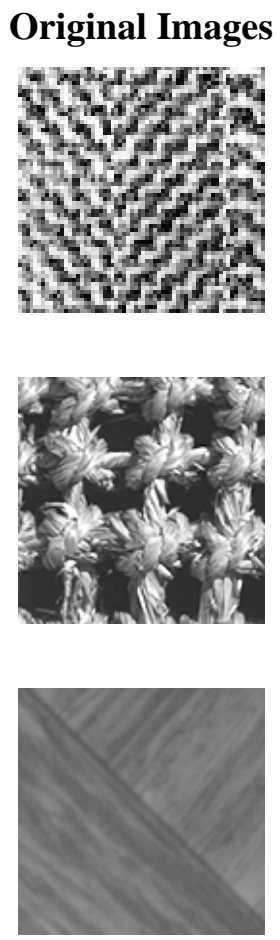
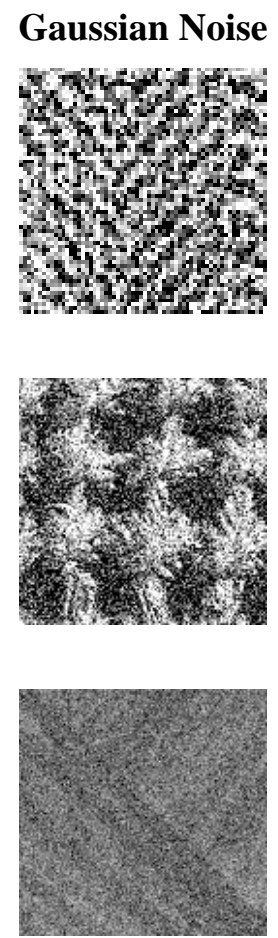

Exponential Noise

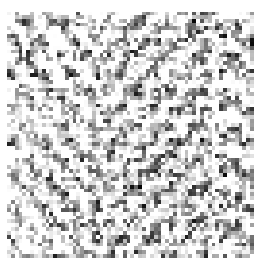

(a)

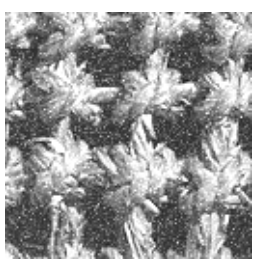

(b)

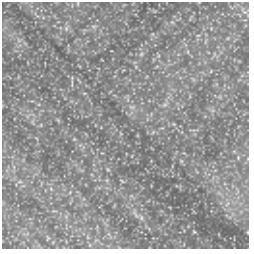

Uniform Noise
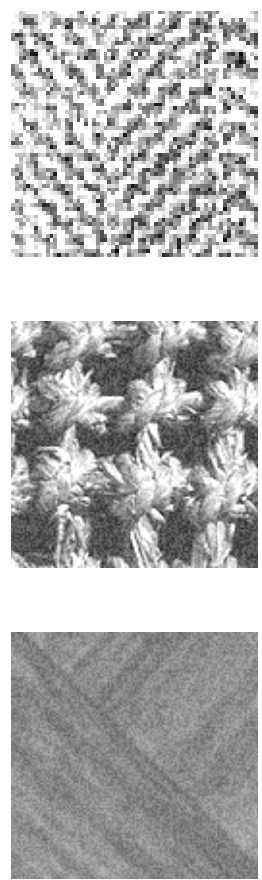

(c)

Figure 4: Example of (a) Brodatz (b) Vistex (c) Outex 13 textures degraded by additive Gaussian Exponential and Uniform noise.

\subsection{Classification of Outex13 textures}

Considering that the Outex 13 dataset consists of colour textures, only their luminance component was used in our study. The computation of the luminance component $Y$ in the case of the Outex 13 color textures has been obtained by the formula $Y=0.6534 R+0.3190 G+0.0277 B$ as suggested in [49], where $R, G$ and $B$ represent the red, green and blue components respectively. 


\begin{tabular}{l|cc}
\hline & $\begin{array}{c}\text { Crisp } \\
\boldsymbol{B P}\end{array}$ & $\begin{array}{c}\text { Fuzzy } \\
\boldsymbol{B P}\end{array}$ \\
\hline $\boldsymbol{L B P}$ & 84.5 & 86.4 \\
\hline $\boldsymbol{L B P} / \boldsymbol{C}$ & 86.3 & 86.5 \\
\hline $\boldsymbol{M B P}$ & 83.4 & 84.8 \\
\hline $\boldsymbol{L E P}$ & 21.9 & 38.8 \\
\hline
\end{tabular}

Table 2: Best classification results obtained for the Outex13 dataset of natural textures without any artificial noise degradation.

The classification experiments performed with the original Outex 13 textures showed that the FBP-based approaches can provide a marginal advantage over the BP-based ones (Table 2). For instance, the use of FLBP instead of LBP features improved accuracy by $1.9 \%$, the use of FMBP instead of MBP features led to a $1.4 \%$ of improvement, whereas the greatest improvement $(16.9 \%)$ was achieved with the FLEP instead of LEP features.

For the Outex13 datasets with noise levels between $6 \mathrm{~dB}$ and $12 \mathrm{~dB}$ SNR the classification error rate reduction (\%) observed between $\mathrm{BP}$ and $\mathrm{FBP}$-based approaches is presented in Table 3 . It can be noticed that the advantage of the FBP-based approaches is clear, because in all noise-degraded datasets, the FBP-based approaches outperformed the respective crisp approaches. In many cases the classification error rate reduction exceeded $22 \%$.

Noise Level (SNR dB)

\begin{tabular}{|c|c|c|c|c|c|c|c|c|c|}
\hline Noise Type & Methods compared & \multicolumn{2}{|c|}{6} & \multicolumn{2}{|c|}{8} & \multicolumn{2}{|c|}{10} & \multicolumn{2}{|c|}{12} \\
\hline \multirow{4}{*}{ Gaussian } & LBP - FLBP & 15.2 & 45.4 & 17.3 & 58.3 & 19.9 & 64.0 & 22.8 & 70.5 \\
\hline & LBP/C - FLBP/FC & 11.8 & 23.6 & 12.7 & 26.1 & 15.1 & 31.1 & 18.0 & 37.0 \\
\hline & MBP - FMBP & 20.2 & 107.0 & 21.8 & 120.0 & 22.2 & 90.0 & 22.5 & 69.3 \\
\hline & LEP - FLEP & 8.8 & 14.0 & 9.8 & 17.5 & 12.1 & 24.2 & 14.3 & 32.1 \\
\hline \multirow{4}{*}{ Exponential } & LBP - FLBP & 8.9 & 8.7 & 9.2 & 7.9 & 10.9 & 8.0 & 13.7 & 9.4 \\
\hline & LBP/C - FLBP/FC & 9.7 & 8.8 & 9.8 & 8.1 & 14.2 & 12.9 & 22.1 & 24.5 \\
\hline & MBP - FMBP & 13.0 & 20.5 & 14.8 & 19.7 & 19.5 & 20.9 & 21.8 & 21.7 \\
\hline & LEP - FLEP & 8.6 & 16.4 & 10.5 & 22.0 & 14.3 & 33.5 & 15.3 & 37.9 \\
\hline \multirow{4}{*}{ Uniform } & LBP - FLBP & 15.2 & 36.6 & 16.3 & 31.4 & 17.7 & 28.2 & 19.5 & 26.4 \\
\hline & LBP/C - FLBP/FC & 12.3 & 21.6 & 13.3 & 18.9 & 14.6 & 17.2 & 16.2 & 16.3 \\
\hline & MBP - FMBP & 21.7 & 49.2 & 22.5 & 45.2 & 23.4 & 42.1 & 24.5 & 39.6 \\
\hline & LEP - FLEP & 13.4 & 27.9 & 13.6 & 28.1 & 13.8 & 28.4 & 13.9 & 28.7 \\
\hline
\end{tabular}

Table 3: Classification experimental results on Outex13 dataset for four noise levels and four BP-based approaches. On the left of each cell there is the classification error rate reduction (\%) between BP and FBPbased approaches. On the right of each cell there is the Chi-square value computed for this experimental setup. The critical value of the Chi-square distribution is equal to 7.87944 . 
The statistical significance of the above experimental results, have been evaluated through the Chi-square test [50]. The research hypothesis was that each FBP based approach has a significantly improved classification accuracy compared with the accuracy of the corresponding BP based approach. The probability of error level has been selected as $p<0.05$, and the degrees of freedom $\mathrm{d} f=1$. For that pair of values $\{p, \mathrm{~d} f\}$, the critical value of the Chi-square distribution is equal to 7.87944. For each of the experiments presented above the computed Chi Square exceeds this critical value, which clearly indicates that these experimental results are statistically significant. The Chi-square values computed for each noise level and for each method are presented in Table 3 with italics.

The overall experimental results are summarized in Table 4. For each pair of BP-FBP based approaches the mean classification rate reduction is presented for all four noise types with the same mean SNR level. Particularly for the case of the FMBP approach the classification error rate reduction was consistent for every type and level of noise tested, reaching up to $22.9 \%$. Additionally for none of the presented methods the classification error rate reduction has not been lower than $9.9 \%$.

In all cases the increase of the computational time between the fuzzy and the corresponding crisp BPbased approaches, ranged between $0.4 \%$ and $14.8 \%$ (Table 5). This rather small increase of the computational time has a significant effect on the classification error rate, which presents a reduction between $8.6 \%$ and $24.5 \%$, resulting in a classification accuracy improvement of $5.4 \%$ to $17.9 \%$.

\begin{tabular}{ccc|c|c|c|}
\multirow{2}{*}{ Methods compared } & \multicolumn{5}{c}{ Noise Level (SNR - dB) } \\
\cline { 2 - 5 } & $\mathbf{6}$ & $\mathbf{8}$ & $\mathbf{1 0}$ & $\mathbf{1 2}$ \\
\hline LBP - FLBP & 13.0 & 14.3 & 16.2 & 18.7 \\
\hline LBP/C - FLBP/FC & 13.1 & 14.4 & 16.5 & 19.0 \\
\hline MBP - FMBP & 18.3 & 19.7 & 21.7 & 22.9 \\
\hline LEP - FLEP & 9.9 & 11.3 & 13.4 & 14.5 \\
\hline
\end{tabular}

Table 4: Mean classification error rate reduction (\%) obtained by each FBP based approach on Outex13 image datasets containing Gaussian, Exponential and Uniform noise.

\begin{tabular}{cc|c|}
\multirow{2}{*}{ Methods compared } & \multicolumn{2}{c}{ Computational Time Increase (\%) } \\
\cline { 2 - 3 } & Minimum & Maximum \\
\hline LBP - FLBP & 0.4 & 0.8 \\
\hline LBP/C - FLBP/FC & 0.6 & 6.5 \\
\hline MBP - FMBP & 3.3 & 14.8 \\
\hline LEP - FLEP & 0.7 & 9.8 \\
\hline
\end{tabular}

Table 5: The mean computational time increase resulted by the fuzzy BP-based approaches compared to their corresponding crisp BP-based approaches. 


\subsection{Classification of Vistex and Brodatz textures}

The classification experiments performed with the original Brodatz and Vistex textures showed that the FBP-based approaches can provide a marginal advantage over the BP-based approaches (Table 6 and Table 7). It should be noted that the absolute accuracy rates in Table 6 and Table 7 are significantly higher than those presented in Table 2, validating that Outex13 is a more challenging dataset. It consists of less diverse textures that are more difficultly discriminated by humans, than the textures of Brodatz and Vistex datasets [42].

As regards the noise-degraded Brodatz and Vistex datasets the classification accuracies, obtained by the FBP-based approaches, showed a consistent improvement over those obtained by the respective BP-based approaches. For both Brodatz and Vistex image datasets, with mean SNR level ranged between $6 \mathrm{~dB}$ to $12 \mathrm{~dB}$, the classification experimental results are presented in Table 8 and Table 9. The mean classification rate reduction observed between each pair of BP-FBP based approaches for Brodatz dataset ranged between $28.0 \%$ and $53.7 \%$ (Table 8 ), and for the Vistex dataset between $7.1 \%$ and $30.7 \%$ (Table 9).

The statistical significance of the experimental results with Brodatz and Vistex datasets has been evaluated through the Chi-square test, in the same way as described in the paragraph 4.1. Although in some of these experiments the computed Chi Square value was higher than the critical value (7.87944), there is also a set of experiments where the computed Chi Square values were lower than the critical value. This indicates that in some cases the experimental results are not statistical significant for the image datasets of Brodatz and Vistex. To be more specific the vast majority of the experimental results from Brodatz images with Gaussian and exponential noise have been found to be statistically significant. On the same time, most of the experimental results from Brodatz images with uniform noise did not prove to be of statistical significance. For the Vistex images with Gaussian noise most of the experimental results are statistically significant, while most of the experimental results from images with uniform and exponential noise did not prove statistically significant.

\begin{tabular}{l|cc}
\hline & $\begin{array}{c}\text { Crisp } \\
\text { BP }\end{array}$ & $\begin{array}{c}\text { Fuzzy } \\
\text { BP }\end{array}$ \\
\hline $\mathbf{L B P}$ & 98.8 & 98.8 \\
\hline $\mathbf{L B P} / \boldsymbol{C}$ & 98.8 & 99.6 \\
\hline $\boldsymbol{M B P}$ & 98.0 & 98.8 \\
\hline $\mathbf{L E P}$ & 94.1 & 95.7 \\
\hline
\end{tabular}

Table 6: Best classification results obtained for the Brodatz dataset of natural textures without any artificial noise degradation.

\begin{tabular}{l|cc}
\hline & $\begin{array}{c}\text { Crisp } \\
\text { BP }\end{array}$ & $\begin{array}{c}\text { Fuzzy } \\
\text { BP }\end{array}$ \\
\hline $\boldsymbol{L B P}$ & 96.1 & 96.1 \\
\hline $\boldsymbol{L B P} / \boldsymbol{C}$ & 96.7 & 97.2 \\
\hline $\boldsymbol{M B P}$ & 93.7 & 93.7 \\
\hline $\boldsymbol{L E P}$ & 89.8 & 90.9 \\
\hline
\end{tabular}

Table 7: Best classification results obtained for the Vistex dataset of natural textures without any artificial noise degradation. 


\begin{tabular}{cc|c|c|c|}
\multirow{2}{*}{ Methods compared } & \multicolumn{5}{c}{ Noise Level (SNR - dB) } \\
\cline { 2 - 5 } & $\mathbf{6}$ & $\mathbf{8}$ & $\mathbf{1 0}$ & $\mathbf{1 2}$ \\
\hline LBP - FLBP & 34.5 & 47.5 & 45.9 & 41.9 \\
\hline LBP/C - FLBP/FC & 41.4 & 49.1 & 49.8 & 53.7 \\
\hline MBP - FMBP & 28.0 & 42.3 & 41.7 & 40.8 \\
\hline LEP - FLEP & 36.5 & 40.9 & 41.6 & 44.3 \\
\hline
\end{tabular}

Table 8: Mean classification error rate reduction (\%) obtained by each FBP based approach on Brodatz image datasets containing Gaussian, Exponential and Uniform noise.

\begin{tabular}{ccc|c|c|c|}
\multirow{2}{*}{ Methods compared } & \multicolumn{5}{c}{ Noise Level (SNR - dB) } \\
\cline { 2 - 5 } & $\mathbf{6}$ & $\mathbf{8}$ & $\mathbf{1 0}$ & $\mathbf{1 2}$ \\
\hline LBP - FLBP & 27.8 & 30.7 & 30.1 & 25.3 \\
\hline LBP/C - FLBP/FC & 19.1 & 23.2 & 26.1 & 24.0 \\
\hline MBP - FMBP & 26.6 & 27.6 & 26.0 & 24.4 \\
\hline LEP - FLEP & 11.9 & 9.4 & 7.7 & 7.1 \\
\hline
\end{tabular}

Table 9: Mean classification error rate reduction (\%) obtained by each FBP based approach on Vistex image datasets containing Gaussian, Exponential and Uniform noise.

\subsection{Segmentation of natural scenes}

In order to validate the advantageous performance of the FBP-based texture representation approaches visually, a number of clustering-based segmentation experiments was conducted with a set of natural scenes from the Vistex image collection [44] . The segmentation task was assigned to the Expectation-Maximization (EM) clustering algorithm, which is unsupervised and generally effective even if the clusters are not hyperspherical [51].

The FLBP/FC texture representation approach, which generally resulted in the highest classification results in the previous paragraph, was considered for these segmentation experiments. Feature extraction was performed in a raster-scanning way using sliding windows of $8 \times 8$ and $16 \times 16$ pixels with a slide step of 1 pixel.

Indicative segmentation results on three Vistex images are illustrated in Fig. 5. These images were selected from the following natural scenes GrassLand/context1, GrassLand2/context2, and GroundWaterCity/context1. Figure 5 shows that the clustering algorithm using the LBP/C features hardly managed to separate the two texture classes of each image, resulting in a visually unsatisfactory segmentation. On the contrary, the quality of the segmentation obtained with the FLBP/FC features is significantly better, as it includes less misclassified regions in all cases. 


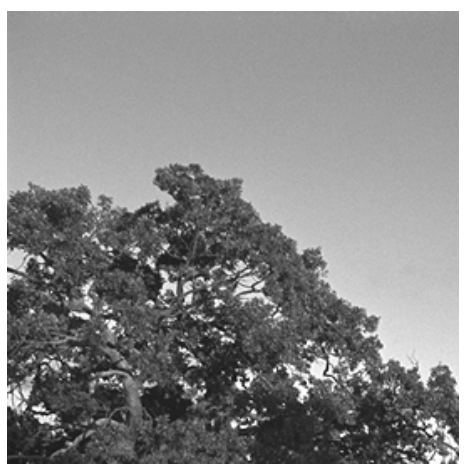

(a)

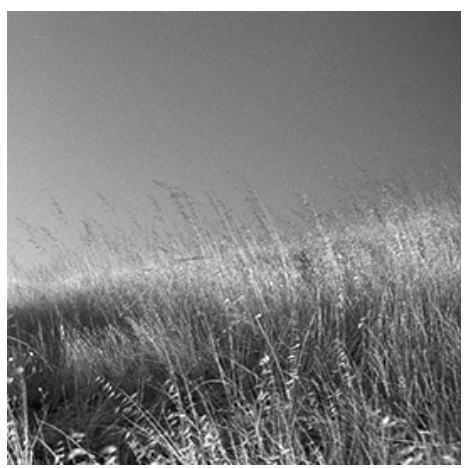

(b)

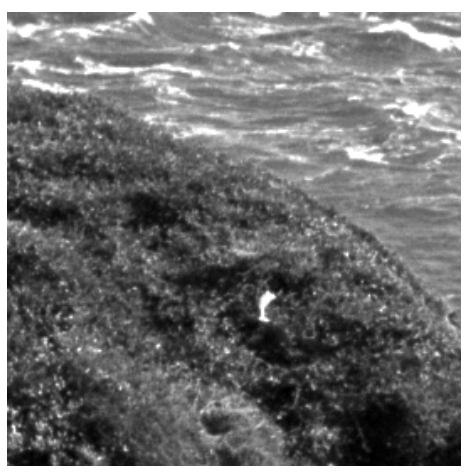

(c)

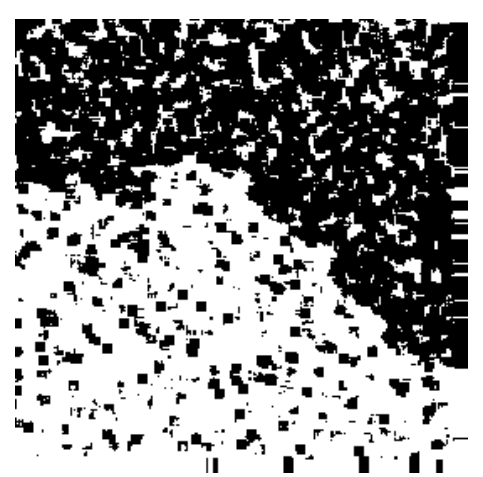

(d)

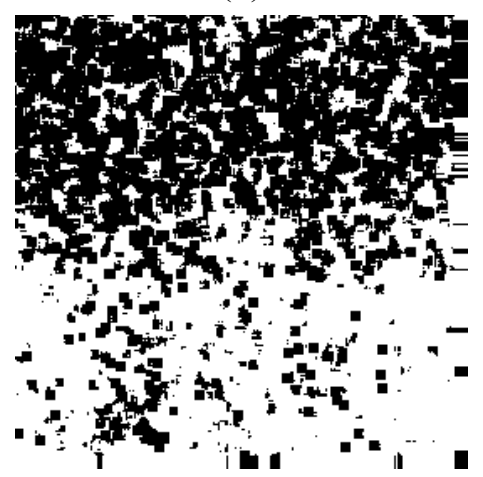

(e)

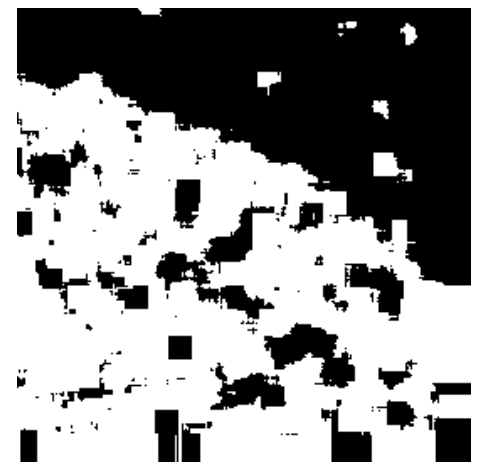

(f)

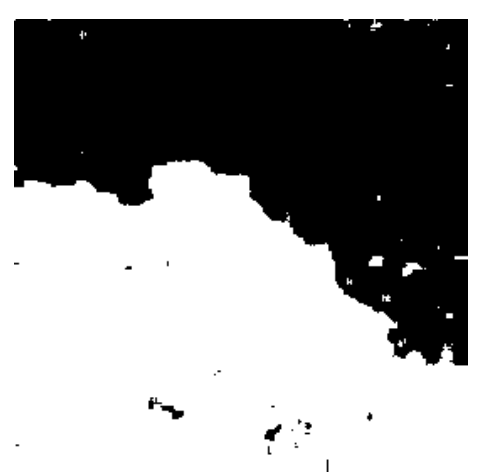

$(\mathrm{g})$

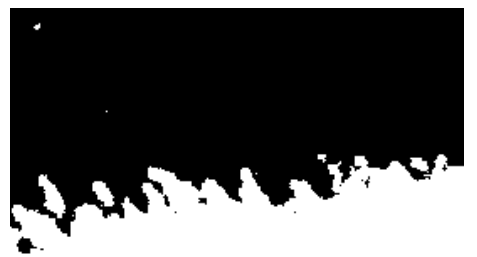

(h)

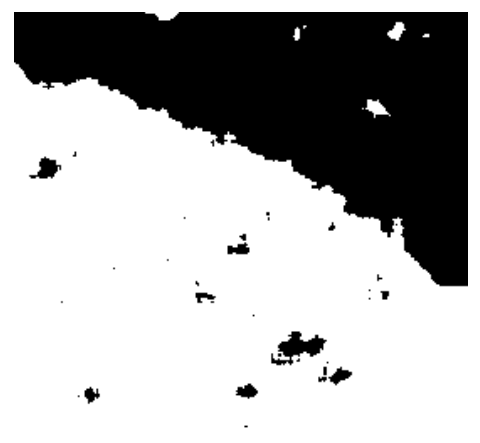

(i)

Figure 5: Vistex natural scenes (a) Grass-Land/context1, (b) GrassLand2/context2, (c) GroundWaterCity/context1 segmented using EM with (d)-(f) LBP/C features and (g)-(i) FLBP/FC features.

\section{Conclusions}

We presented an uncertainty-aware methodology for the extraction of BP-based textural features using fuzzy logic. The proposed methodology is generic and can be applied on BP-based texture representation approaches, to improve their robustness against noise. In this study we applied it on four representative BPbased approaches, namely the LBP, LBP/C, MBP and LEP, and derived the FLBP, FLBP/FC, FMBP and FLEP feature sets. The results of their experimental evaluation on reference datasets lead to the following conclusions:

- the FBP-based approaches provide more discriminative representation of natural textures than the crisp BP-based approaches; 
- the FBP texture representation is more tolerant to white Gaussian, Exponential and Uniform additive noise, whereas the highest tolerance has been observed with the Gaussian noise;

- the fuzzification parameter value should be higher for images with lower SNR;

- the advantage of the FBP over the crisp BP approaches becomes more evident for moderate noise levels;

- in most classification experiments the best accuracy has been achieved with the FLBP/FC approach;

- the improved texture discrimination capability of the FLBP/FC approach was qualitatively validated with unsupervised segmentation of natural scenes;

- the computational cost of the FBP-based approaches has been close to that of the BP-based approaches;

- the Chi-square test proved the statistical significance of the experimental results presented on the most challenging Outex13 dataset;

Future perspectives of this work include the utilization of FBP-based approaches for texture representation in various applications involving texture recognition in low SNR environments.

\section{Software availability}

Implementations of the FLBP, FLBP/FC, FLEP, FMBP approaches are publicly available for download at http://rtsimage.di.uoa.gr/index.php?option=com_content\&view=article\&id=75\&Itemid=69.

\section{Acknowledgment}

This work was supported by the Greek General Secretariat of Research and Technology (25\%), the European Social Fund (75\%), and the private sector, under the framework of Measure 8.3 of E.P. Antagonistikotita - 3rd European Support Framework - PENED 2003 (grant no. 03-ED-662).

\section{References}

[1] G. Castellano, L. Bonilha, L.M. Li, F. Cendes, "Texture analysis of medical images", Clinical Radiology, 59 (2004) pp. 1061-1069.

[2] O. Tsymbal, V. Lukin, N. Ponomarenko, A. Zelensky, K. Egiazarian, J. Astola, "Three-State Locally Adaptive Texture Preserving Filter for Radar and Optical Image Processing", EURASIP Journ. on Ap. Sign. Proc., 8 (2005) 1185-1204.

[3] Y. Li, J.X. Peng, "Remote Sensing Texture Analysis Using Multi-Parameter and Multi-Scale Features", Photo. Engin. \& Remote Sens., 69 (2003) 351-356.

[4] Z. Wang, J.H. Yong, "Texture Analysis and Classification With Linear Regression Model Based on Wavelet Transform", IEEE Transactions on Image Processing, (2008) 1421-1430.

[5] T. Wagner, Texture Analysis, "Handbook of Computer Vision and Application", Academic Press, 1999.

[6] M. Petrou, P. G. Sevilla, "Image Processing: Dealing With Texture”, New York: Wiley, 2006. 
[7] T. Ojala, M. Pietikäinen, D. Harwood, “A comparative study of texture measures with classification based on featured distribution", Pattern Recognition, 29 (1996) 51-59.

[8] C. Chan, J. Kittler, K. Messer, "Multi-Scale Local Binary Pattern Histograms for Face Recognition", ICB 2007, Seoul, Korea, LNCS 4642, Springer, 809-818 (2007).

[9] G. Pok, K. Ryu, J. Lyu, "Rotation and Gray-Scale Invariant Classification of Textures Improved by Spatial Distribution of Features", DEXA 2005, Copenhagen, Denmark, LNCS 3588 (2005) 250-259.

[10] J. Fehr, "Rotational Invariant Uniform Local Binary Patterns For Full 3D Volume Texture Analysis", FINSIG 2007, Oulu, Finland (2007).

[11] W. Weixin, L. Jianguo, W Wang, Y. Zhang, "Markov Chain Local Binary Pattern and Its Application to Video Concept Detection", IEEE International Conference on Image Processing, (2008).

[12] L. Ma, R.C. Staunton, "Optimum Gabor filter design and local binary patterns for texture segmentation", Pattern Recognition Letters, 29 (2008) 664-672.

[13] Y. Cheng-Hao, C. Shu-Yuan, "Retrieval of translated, rotated and scaled color textures", Pattern Recognition 36 (2003) 913 - 929.

[14] A. Hafiane, G. Seetharaman, B. Zavidovique, "Median binary pattern for textures Classification", ICIAR, (2007) 387-398.

[15] A. Hafiane, G. Seetharaman, K. Palaniappan, B. Zavidovique, "Rotationally Invariant Hashing of Median Binary Patterns for Texture Classification", Proc. Image Analysis and Recognition, LNCS, (2008) 619-629.

[16] J. Iivarinen, "Surface defect detection with histogram-based texture features", Intelligent Robots and Computer Vision XIX: Algorithms, Techniques, and Active Vision, 4197, (2000) 140-145.

[17] O. Silven, M. Niskanen, H. Kauppinen, "Wood inspection with non-supervised clustering", Machine Vision and Applications, 13 (2003) 275-285.

[18] F. Tajeripour, E. Kabir, A. Sheikhi, "Fabric Defect Detection Using Modified Local Binary Patterns", EURASIP Journal on Advances in Signal Processing, (2008).

[19] H. Hadizadeh, S Baradaran Shokouhi, "Random Texture Defect Detection Using 1-D Hidden Markov Models Based on Local Binary Patterns", IEICE Transactions on Information and Systems, (2008) 1937-1945.

[20] H. Grabner, TT. Nguyen, B. Gruber, H. Bischof, "On-line boosting-based car detection from aerial images", ISPRS Journal of Photogrammetry and Remote Sensing, 63 (2008) 382-396.

[21] C-J. Liao, S-Y. Chen, “Complementary retrieval for distorted images", Pattern Recognition, 35 (2002) 1705-1722.

[22] J. Jiang, A. Armstrong, GC Feng, "Web-based Image Indexing and Retrieval in JPEG compressed domain", Multimedia Systems, 9 (2004) 424-432.

[23] D. Grangier, S. Bengio, "A Discriminative Kernel-Based Approach to Rank Images from Text Queries”, IEEE Transactions on Pattern Analysis and Machine Intelligence, 30 (2008) 1371-1384.

[24] Z. Stan, C. RuFeng, L. ShengCai, Z Lun, "Illumination Invariant Face Recognition Using NearInfrared Images", IEEE Transactions on Pattern Analysis and Machine Intelligence, 29 (2007) 627639.

[25] X. Zhang, Y. Gao, M. Leung, "Recognizing Rotated Faces From Frontal and Side Views: An Approach Toward Effective Use of Mugshot Databases", IEEE Transactions on Information Forensics and Security, 3 (2008) 684-697.

[26] A. Destrero, C. De Mol, F. Odone, A. Verri, "A Regularized Framework for Feature Selection in Face Detection and Authentication", International Journal of Computer Vision, (2008).

[27] C. Shan, S. Gong, P. McOwan, "Facial Expression Recognition Based on Local Binary Patterns: A Comprehensive Study", Image and Vision Computing, (2008).

[28] D.K. Iakovidis, D. Maroulis, S. Karkanis, "An intelligent system for automatic detection of gastrointestinal adenomas in video endoscopy", Computers in Biology and Medicine, 36 (2006) 10841103. 
[29] L. Nanni, A. Lumini, “A reliable method for cell phenotype image classification", Artificial Intelligence in Medicine, 43 (2008) 87-97.

[30] O. Sertel, J. Kong, H. Shimada, U.V. Catalyurek, J.H. Saltz, M.N. Gurcan, “Computer-aided prognosis of neuroblastoma on whole-slide images: Classification of stromal development", Pattern Recognition, 42 (2009) 1093-1103.

[31] R. Garcia, X. Cufi, J. Batlle, "Detection of matchings in a sequence of underwater images through texture analysis", Proc. IEEE International Conference on Image Processing, 1 (2001) 361-364.

[32] M. Heikkila, M. Pietikainen, "A texture-based method for modeling the background and detecting moving objects", IEEE Transactions on Pattern Analysis and Machine Intelligence, 28 (2006) 657662.

[33] N. Petrovic, L. Jovanov, A. Pizurica, W. Philips, "Object Tracking Using Naive Bayesian Classifiers", Proc. Advanced Concepts for Intelligent Vision Systems, LNCS, 5259 (2008) 775-784.

[34] R.C. Gonzalez, R.E. Woods, "Digital Image Processing”, Pearson Education, 2001.

[35] A. Barcelo, E. Montseny, and P. Sobrevilla, "Fuzzy Texture Unit and Fuzzy Texture Spectrum for texture characterization", Fuzzy Sets Syst. 158, 3 (2007) 239-252.

[36] E.G. Keramidas, "Ultrasound Image Processing and Analysis Framework", MSc thesis, University of Athens, Greece (2007).

[37] T. Ahonen, M. Pietikäinen, "Soft histograms for local binary patterns", Finnish Signal Processing Symposium, (2007).

[38] E.G. Keramidas, D.K. Iakovidis, D. Maroulis, N. Dimitropoulos, "Thyroid Texture Representation via Noise Resistant Image Features", IEEE International Symposium on Computer-Based Medical Systems, (2008) 560-565.

[39] E.G. Keramidas, D. Iakovidis, D. Maroulis, "Noise Robust Statistical Feature Distributions for Texture Analysis", European Signal Processing Conference (2008).

[40] L. Zadeh, "Fuzzy Sets", Journal of Information and Control, 8 (1965) 338-353.

[41] M. Petrou, P. Bosdogianni, Image Processing: The Fundamentals, John Wiley\& Sons, 1999.

[42] Outex13 Texture classification test suite. University of Oulu, http://www.outex.oulu.fi/index.php?page=classificati-on\#Outex_TC_00013, 2007.

[43] P. Brodatz, "Texture: A Photographic Album for Artists and Designers", Dover Publications, New York, USA, 1996.

[44] MIT MediaLab, VisTex Database, http://vismod.media.mit.edu/ vismod/imagery/ VisionTexture/vistex.html, 2002.

[45] S. Theodoridis, K. Koutroumbas, Pattern Recognition, Academic Press, 2008.

[46] B.D. Ripley, Pattern Recognition and Neural Networks, Cambridge University Press, 1996

[47] M.J. Swain, D.H. Ballard, "Color indexing”, International .Journal of Computer Vision, 7 (1991) 1132.

[48] I. Pitas, "Digital Image Processing Algorithms", Prentice Hall International Series in Acoustics, 1995.

[49] R.G. Hunt, Measuring Colour, Fountain Press, England, Third ed., 1998.

[50] E. Fienberg, "The Analysis of Cross-Classified Categorical Data", Springer, (2007), ISBN 0387728244.

[51] A. Dempster, N. Laird, D. Rubin, "Maximum Likelihood from Incomplete Data via the EM Algorithm", J. Royal Statistical Soc., 39 (1977) 1-38. 